\title{
EFFECTS OF IRON SUPPLAMENTATION ON RBC PARAMETERS AND INDICES, PERFORMANCE AND HEALTH IN NEONATAL DAIRY CALVES
}

\author{
M. Mohri, H. A. Seifi, F. Sarrafzadeh \\ Department of Clinical Sciences, School of Veterinary Medicine, Ferdowsi University of Mashhad, \\ Tel: 0098511 6610430, Fax: 0098511 6620166, e mail: mohri@kamal.um.ac.ir, Mashhad, Iran
}

Iron deficiency is usually primary and most likely to occure in newborn animals whose sole source of iron is the milk of the dam, milk being a poor source of iron. There have been several studies of iron deficiency anemia and effects of iron supplementation in veal calves but information concerning to iron status and iron supplementation in neonatal dairy calves (Heifer replacement system) are limited.

In order to evaluate the effects of oral iron supplementation on RBC parameters (RBC number, Packed cell volume and Hemoglobin concentration) and indices (MCV, MCHC), performance (Weight, height, length) and health in neonatal dairy calves a case control study was performed. 40 such calves were considered and divided at random into two groups: test (20) and control (20). The dietary and other management factors were identical for all the calves. The Calves were separated from dams immediately after birth and fed dams colostrums for two days and herd milk until 10 days of life and then concentrate and alfa alfa were added to the diet. In the test group oral iron as ferrous sulfate was supplemented to each calf at a rate of $150 \mathrm{mg} /$ daily for 28 days.

Sampling was conducted from jugular vein immediately after birth (before iron supplementation) and days 7, 14, 21 and 28. The blood was added to EDTA containing tube for hematological measurements and plane tube for iron and TIBC measurements. The levels of RBC number (manual standard method), PCV (microhematocrite), hemoglobin concentration (cyanmethemoglobin method) and indices (calculated), serum iron (colorimetric method), TIBC (colorimetric method), TIBC saturation (calculated), body weight, height, length; total, weekly and daily weight gain and disease occurrence were recorded and compared by appropriate statistical methods (parametric independent $t$ test and chi square analysis) between groups. $\mathrm{P}<0.05$ was considered as significant.

The hemoglobin, PCV, serum iron and TIBC saturation levels were significantly higher at the end of second, third, and fourth weeks of life in the test group $(p<0.05)$. Higher level of RBC in the test group was seen only at the end of fourth week $(p<0.05)$. Weight gain during fourth week was significantly higher in the test group $(\mathrm{p}<0.05)$. There was significantly lower TIBC value at the end of fourth week in the test group $(\mathrm{p}<0.05)$. Marginal differences were seen for total weight gain and daily weight gain between groups $(\mathrm{p}=0.05)$. No significant differences were seen for other parameters and health between groups.

In conclusion, it seems the amount of iron uptake by neonatal dairy calves is insufficient at least during first month of life and iron supplementation has beneficial effects on RBC parameters and performance.

\section{Reference}

Lindt, F; Blum, J. W. Occurrence of iron deficiency in growing cattle. J Vet Med (A) 1994, 41: 237-246 